Research Article

\title{
Exact Solution for the Torsional Vibration of an Elastic Pile in a Radially Inhomogeneous Saturated Soil
}

\author{
Xibin Li $\mathbb{D},{ }^{1}$ Zhiqing Zhang $\mathbb{D},{ }^{1}$ and Jianchao Sheng ${ }^{2}$ \\ ${ }^{1}$ School of Landscape Architecture, Zhejiang A \& F University, Hangzhou, Zhejiang 311300, China \\ ${ }^{2}$ School of Civil Engineering, Shaoxing University, Shaoxing, Zhejiang 312000, China \\ Correspondence should be addressed to Zhiqing Zhang; zhangzhiqing2000@163.com
}

Received 20 November 2020; Revised 23 December 2020; Accepted 11 January 2021; Published 25 January 2021

Academic Editor: Zhaoqing Wang

Copyright (ㅇ 2021 Xibin Li et al. This is an open access article distributed under the Creative Commons Attribution License, which permits unrestricted use, distribution, and reproduction in any medium, provided the original work is properly cited.

An exact solution is proposed to study the time-harmonic torsional vibration of an elastic pile embedded in a radially inhomogeneous saturated soil. The radially inhomogeneous saturated soil is composed of inner disturbed and outer semi-infinite undisturbed concentric annular regions, with the shear modulus of the inner region changing in an exponential form along the radial direction. The governing equation of each region of the saturated soil is solved through rigorous mathematical derivation and the soil torsional impedance is derived with an exact and explicit expression. Making use of the boundary and continuity conditions of the pile-soil system, the torsional complex stiffness at the pile top is obtained in an exact closed form in the frequency domain. Selected numerical results are presented to investigate the influence of the radial inhomogeneity of the surrounding soil on the vibration characteristics of the pile-soil system.

\section{Introduction}

Soil-structure dynamic interaction problem, including the interaction between a loaded rigid disc (footing) and the adjacent soil and the interaction between the pile and surrounding soil, has been the subject of extensive studies in civil engineering for many years. For these interaction problems, the establishment of a dynamic model of the soil medium and the corresponding solution is the key to study the vibration characteristics of the soil-structure system. For instance, Pan et al. [1] and Zhang and Pan [2] used fundamental solutions of the surface/buried annular patch torsional load on/in the soil medium and then solved the dynamic impedance of the rigid circular disc via integral least-square approach. Pak and Abedzadeh [3] used boundary integral technique to determine the reaction of the soil and finally proposed the solution for the rigid circular disc under time-harmonic torsional load. For the pile-soil dynamic interaction problem, the analytical method $[4,5]$ and numerical method [6] are developed to solve the torsional vibration of an elastic pile embedded in a homogeneous or layered soil medium.
It should be pointed out that when studying pile-soil torsional vibration problems, the surrounding soil was mostly regarded as a radially uniform medium. However, in actual engineering, no matter precast piles or cast-in-place piles, they are bound to disturb or squeeze the surrounding soil in a certain range during the construction process. Therefore, the properties of the soil around the pile body (e.g., shear modulus of the soil) inevitably have continuous variation, so the soil shows obvious radial inhomogeneity. In an early study, Novak and Sheta [7] proposed a radially inhomogeneous medium model based on the assumption of plane strain and analyzed the torsional and longitudinal impedance of the weakened soil in the inner region induced by the construction of the pile. Afterwards, researchers conducted detailed analyses on the torsional and longitudinal vibration characteristics of the radially inhomogeneous soils. However, these studies regarded the foundation soil as a single-phase medium [8-13].

It is worth noting that saturated soil is very common in engineering practice. Since Biot $[14,15]$ established the theory of wave propagation in fluid-saturated porous medium, this theory has been widely used in engineering. Li 
et al. [16] studied the torsional vibration of an elastic pile embedded in an inhomogeneous saturated soil, but the solution is actually semianalytical due to the fact that the surrounding soil is subdivided into many sublayers to simulate the continuous change of soil properties. Considering the deficiencies of previous research, the objective of the present study is to propose an exact solution to study the time-harmonic torsional vibration of an elastic pile embedded in a radially inhomogeneous saturated soil with soil modulus changing in an exponential form along the radial direction. Based on the derived solution, the influence of the characteristic parameters of the radially inhomogeneous soil on the torsional impedance of the soil and the torsional complex stiffness at the pile top is analyzed in detail. The exact solution presented in this study can be served as benchmarks for future numerical simulations.

\section{Statement of the Boundary-Value Problem}

2.1. Geometric Model and Assumptions. The problem to be solved is illustrated in Figure 1, where an elastic circular pile of radius $r_{0}$ and length $H$ is embedded in a radially inhomogeneous saturated soil. The elastic pile is subjected to a time-harmonic torsional load $T_{0} e^{i \omega t}$, in which $\omega$ and $t$ are the circular frequency of excitation and time variable, respectively. In view of the symmetry of the problem, the cylindrical coordinate system $(r, \theta, z)$ is attached to the saturated soil layer with its origin at the center of the pile top and on the surface of soil layer. Considering the influence of construction disturbance in pile driving process, the surrounding saturated soil can be modeled as two concentric annular regions: one is a disturbed soil region with a radial thickness $t_{m}$, and the other is a radially semi-infinite, undisturbed uniform soil region. The distance from the interface of the two regions to the center of the pile is $R_{0}$. In order to establish a mathematical model for this problem, it is assumed that the pile is well bonded with the surrounding soil, and the interface between two regions is also in perfect contact. Based on the study by Dotsos and Veletsos [10], the shear modulus of the soil in the inner disturbed region is assumed to change in an exponential form along the radial direction.

In order to simulate the exponential change of the shear modulus of the soil in the inner region along the radial direction (see Figure 2), the complex shear modulus of the soil in the inner and outer regions can be expressed as

$$
G_{s}^{*}(r)= \begin{cases}G_{s m}\left(1+i D_{s m}\right)\left(r / r_{0}\right)^{m}, & \left(r_{0} \leq r \leq R_{0}\right), \\ G_{s 0}\left(1+i D_{s 0}\right), & \left(r \geq R_{0}\right),\end{cases}
$$

where $G_{s 0}$ and $G_{s m}$ are, respectively, the shear moduli of the soil in the outer undisturbed region and at the pile-soil interface; $D_{s 0}$ and $D_{s m}$ are the corresponding soil damping coefficients, respectively; $i=(-1)^{0.5} ; m$ is a real number with $m \neq 1$ representing the continuous change of shear modulus in an exponential form and $m=1$ representing the continuous change of shear modulus in a reduced linear form.

Considering the continuous change of the soil shear modulus and according to equation (1), the following relationship exists at the interface between the inner and outer soil regions:

$$
G_{s m}\left(\frac{R_{0}}{r_{0}}\right)^{m}=G_{s 0}
$$

2.2. Governing Equation. According to the dynamic consolidation theory proposed by Biot $[14,15]$, the equation of motion of the soil under dynamic torsional load can be written as

$$
\frac{\partial \sigma_{r \theta}}{\partial r}+\frac{\partial \sigma_{\theta z}}{\partial z}+2 \frac{\sigma_{r \theta}}{r}=\rho \frac{\partial^{2} u_{\theta}(r, z, t)}{\partial t^{2}}+\rho_{f} \frac{\partial^{2} w_{\theta}(r, z, t)}{\partial t^{2}}
$$

where $u_{\theta}(r, t)$ and $w_{\theta}(r, t)$ are, respectively, the circumferential displacement of the solid phase and that of the fluid phase relative to the solid phase; $\sigma_{i j}(i, j=r, \theta, z)$ represents the total stress components; $\rho_{s}$ and $\rho_{f}$ are the densities of soil phase and pore fluid, respectively; $\rho=(1-n) \rho_{s}+n \rho_{f}$ is the density of the soil, with $n$ being the porosity of the soil.

Militano and Rajapakse [17] pointed out that neglecting the gradient of the soil stress component along $z$ direction has negligible influence on the dynamic response of the pile. Therefore, under the same assumptions and considering the continuous change of the shear modulus of the soil, the equation of motion of the soil can be further expressed as

$$
\begin{aligned}
G_{s}^{*}(r)\left(\frac{\partial^{2} u_{\theta}(r, t)}{\partial r^{2}}+\frac{1}{r} \frac{\partial u_{\theta}(r, t)}{\partial r}-\frac{u_{\theta}(r, t)}{r^{2}}\right) & +\frac{d G_{s}^{*}(r)}{d r}\left(\frac{\partial u_{\theta}(r, t)}{\partial r}-\frac{u_{\theta}(r, t)}{r}\right) \\
& =\rho \frac{\partial^{2} u_{\theta}(r, t)}{\partial t^{2}}+\rho_{f} \frac{\partial^{2} w_{\theta}(r, t)}{\partial t^{2}} .
\end{aligned}
$$




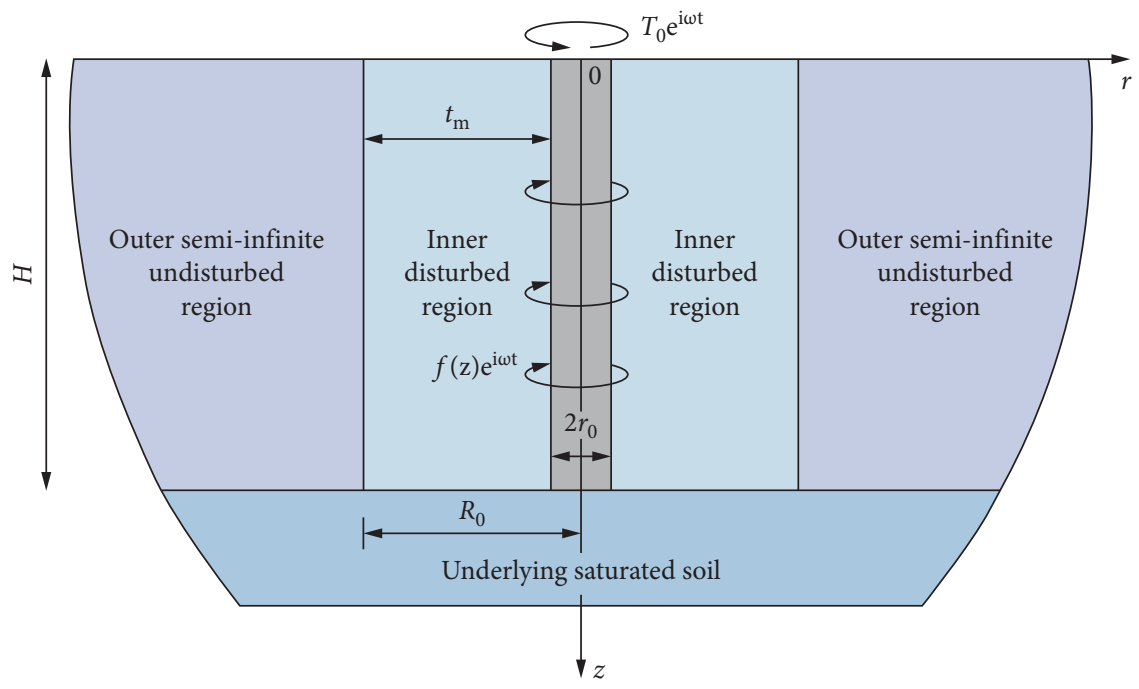

FIgURE 1: Dynamic torsional interaction between an elastic pile and a radially inhomogeneous saturated soil.

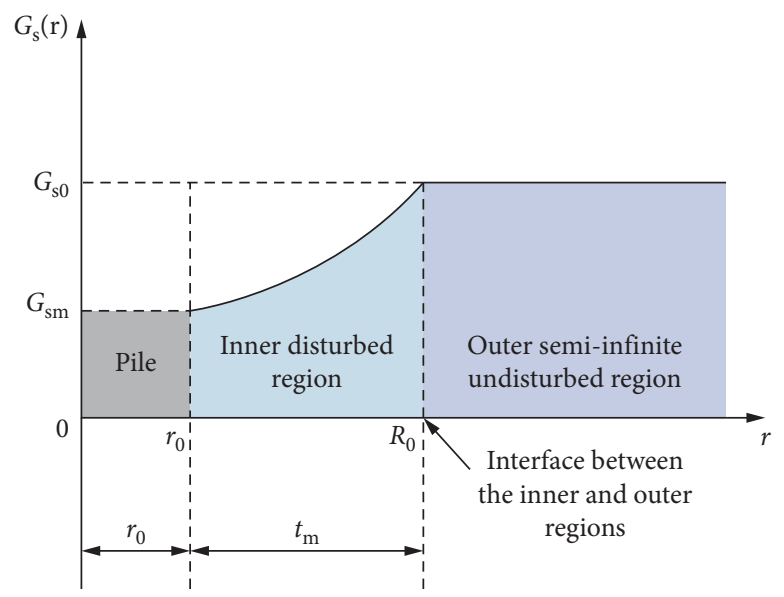

FIGURE 2: Diagrammatic sketch of the radially inhomogeneous soil.

Substituting equation (1) into equation (4), we have

$$
\begin{aligned}
& G_{s m}\left(1+i D_{s m}\right)\left(\frac{r}{r_{0}}\right)^{m}\left(\frac{\partial^{2} u_{\theta}(r, t)}{\partial r^{2}}+\frac{1}{r} \frac{\partial u_{\theta}(r, t)}{\partial r}-\frac{u_{\theta}(r, t)}{r^{2}}\right)+\frac{m G_{s m}\left(1+i D_{s m}\right)}{r_{0}}\left(\frac{r}{r_{0}}\right)^{m-1}\left(\frac{\partial u_{\theta}(r, t)}{\partial r}-\frac{u_{\theta}(r, t)}{r}\right) \\
& =\rho_{m} \frac{\partial^{2} u_{\theta}(r, t)}{\partial t^{2}}+\rho_{f m} \frac{\partial^{2} w_{\theta}(r, t)}{\partial t^{2}}, \quad\left(r_{0} \leq r \leq R_{0}\right), \\
& G_{s 0}\left(1+i D_{s 0}\right)\left(\frac{\partial^{2} u_{\theta}(r, t)}{\partial r^{2}}+\frac{1}{r} \frac{\partial u_{\theta}(r, t)}{\partial r}-\frac{u_{\theta}(r, t)}{r^{2}}\right)=\rho_{0} \frac{\partial^{2} u_{\theta}(r, t)}{\partial t^{2}}+\rho_{f 0} \frac{\partial^{2} w_{\theta}(r, t)}{\partial t^{2}}, \quad\left(r \geq R_{0}\right),
\end{aligned}
$$

where $\quad \rho_{m}=\left(1-n_{m}\right) \rho_{s m}+n_{m} \rho_{f m} \quad$ and $\rho_{0}=\left(1-n_{0}\right) \rho_{s 0}+n_{0} \rho_{f 0}$ are the densities of soil in disturbed and undisturbed regions, respectively; $\rho_{s m}\left(\rho_{s 0}\right), \rho_{f m}\left(\rho_{f 0}\right)$, and $n_{\mathrm{m}}\left(n_{0}\right)$ are the densities of solid phase and fluid phase and porosities in disturbed (and undisturbed) regions, respectively.

The fluid motion equation of the saturated soil medium can be expressed in the following simplified form [18, 19]: 


$$
\begin{aligned}
& \frac{\rho_{f m} g}{k_{d m}} \frac{\partial w_{\theta}(r, t)}{\partial t}+\rho_{f m} \frac{\partial^{2} u_{\theta}(r, t)}{\partial t^{2}}+\frac{\rho_{f m}}{n_{m}} \frac{\partial^{2} w_{\theta}(r, t)}{\partial t^{2}}=0, \quad\left(r_{0} \leq r \leq R_{0}\right), \\
& \frac{\rho_{f 0} g}{k_{d 0}} \frac{\partial w_{\theta}(r, t)}{\partial t}+\rho_{f 0} \frac{\partial^{2} u_{\theta}(r, t)}{\partial t^{2}}+\frac{\rho_{f 0}}{n_{0}} \frac{\partial^{2} w_{\theta}(r, t)}{\partial t^{2}}=0, \quad\left(r \geq R_{0}\right), \\
& \quad G_{p} \frac{\partial^{2}}{\partial z^{2}}\left[\phi(z) e^{i \omega t}\right]+\frac{4 f(z) e^{i \omega t}}{r_{0}^{2}}=\rho_{p} \frac{\partial^{2}}{\partial t^{2}}\left[\phi(z) e^{i \omega t}\right],
\end{aligned}
$$

where $k_{d m}$ and $k_{d 0}$ are the horizontal dynamic permeability coefficients of saturated soil in disturbed and undisturbed regions, respectively, and $g$ is the acceleration of gravity.

Due to the time-harmonic vibration of the pile-soil system, $u_{\theta}(r, t)$ and $w_{\theta}(r, t)$ can be further expressed as

$$
\left\{\begin{array}{l}
u_{\theta}(r, t)=u_{\theta}(r) e^{i \omega t}=u_{\theta} e^{i \omega t}, \\
w_{\theta}(r, t)=w_{\theta}(r) e^{i \omega t}=w_{\theta} e^{i \omega t},
\end{array}\right.
$$

where $u_{\theta}=u_{\theta}(r)$ and $w_{\theta}=w_{\theta}(r)$ are, respectively, the amplitude of circumferential displacement of the solid phase and that of the relative circumferential displacement of the fluid phase to the solid phase.

Combining equations (5)-(8) and introducing $\xi=r / r_{0}$, the governing equation for the radially inhomogeneous saturated soil can be expressed as

$$
\begin{aligned}
& \xi^{2} \frac{d^{2} u_{\theta}}{d \xi^{2}}+\xi(1+m) \frac{d u_{\theta}}{d \xi}-\left(1+m+\lambda_{m}^{2} \xi^{2-m}\right) u_{\theta}=0, \quad\left(1 \leq \xi \leq \xi_{0}\right), \\
& \xi^{2} \frac{d^{2} u_{\theta}}{d \xi^{2}}+\xi \frac{d u_{\theta}}{d \xi}-\left(1+\lambda_{0}^{2} \xi^{2}\right) u_{\theta}=0, \quad\left(\xi \geq \xi_{0}\right),
\end{aligned}
$$

where

$$
\begin{aligned}
& b_{m}=\frac{n_{m} \rho_{f m} g}{k_{d m}} \\
& b_{0}=\frac{n_{0} \rho_{f 0} g}{k_{d 0}} \\
& \lambda_{m}=i \omega r_{0}\left[\frac{1}{G_{s m}\left(1+i D_{s m}\right)}\left(\rho_{m}+\frac{n_{m} \rho_{f m} \omega}{\left(i b_{m} / \rho_{f m}\right)-\omega}\right)\right]^{(1 / 2)}, \\
& \lambda_{0}=i \omega r_{0}\left[\frac{1}{G_{s 0}\left(1+i D_{s 0}\right)}\left(\rho_{0}+\frac{n_{0} \rho_{f 0} \omega}{\left(i b_{0} / \rho_{f 0}\right)-\omega}\right)\right]^{(1 / 2)} \\
& \xi_{0}=\frac{R_{0}}{r_{0}} .
\end{aligned}
$$

Given that the elastic pile is forced to vibrate under the action of the time-harmonic torsional load at the pile top, the equation of motion of pile can be expressed as where $\rho_{p}, G_{p}$, and $r_{0}$ are the density, shear modulus, and radius of the pile, respectively, and $f(z)$ is the amplitude of the circumferential shear stress at the pile-soil interface.

2.3. Boundary and Continuity Conditions of Pile-Soil System. Given that the displacement of the soil at infinity tends to zero, the following relationship holds:

$$
u_{\theta}(r \longrightarrow \infty)=0 .
$$

It is assumed that the soil at the interface between inner and outer soil regions (i.e., $r=R_{0}$ ) is well bonded. Then, continuity conditions at $r=R_{0}$ can be written as

$$
\begin{gathered}
u_{\theta}\left(r=R_{0-}\right)=u_{\theta}\left(r=R_{0+}\right), \\
\tau_{r \theta}\left(r=R_{0-}\right)=\tau_{r \theta}\left(r=R_{0+}\right) .
\end{gathered}
$$

The boundary conditions of the pile can be expressed as

$$
\left.\frac{\mathrm{d} \phi(z)}{\mathrm{d} z}\right|_{z=0}=-\frac{T_{0}}{G_{p} I_{p}},
$$

$$
\left.\left[\frac{\mathrm{d} \phi(z)}{\mathrm{d} z}+\frac{\phi(z) k_{p b}}{G_{p} I_{p}}\right]\right|_{z=H}=0,
$$

where $I_{p}=\left(\pi r_{0}^{4} / 2\right)$ is the polar moment of inertia of the pile. It is noted that it is reasonable to use the static stiffness of the disc with the same radius on the surface of the elastic halfspace to simulate the stiffness of the pile bottom [17]. That is to say, the supporting stiffness coefficient $k_{p b}$ of the pile bottom is taken as $\left(16 G_{s b} r_{0}^{3} / 3\right)$ with $G_{s b}$ being the shear modulus of the soil at the bottom of the pile.

The continuity conditions between pile and soil interface can be expressed as

$$
\begin{aligned}
\left.u_{\theta}\right|_{r=r_{0}} & =\phi(z) r_{0}, \\
\left.\tau_{r \theta}\right|_{r=r_{0}}=\left.G_{s m}\left(1+i D_{s m}\right)\left(\frac{\mathrm{d} u_{\theta}}{\mathrm{d} r}-\frac{u_{\theta}}{r}\right)\right|_{r=r_{0}} & =f(z) .
\end{aligned}
$$

\section{Solution of the Pile-Soil System}

When $m \neq 2$, the solutions of equation (9) can be expressed as

$$
\begin{aligned}
& u_{\theta}=\xi^{(-m / 2)}\left[A_{1} K_{2 \kappa-1}\left(\kappa \lambda_{m} \xi^{(1 / \kappa)}\right)+B_{1} I_{2 \kappa-1}\left(\kappa \lambda_{m} \xi^{(1 / \kappa)}\right)\right], \quad\left(1 \leq \xi \leq \xi_{0}\right) \\
& u_{\theta}=C_{1} K_{1}\left(\lambda_{0} \xi\right)+D_{1} I_{1}\left(\lambda_{0} \xi\right), \quad\left(\xi \geq \xi_{0}\right)
\end{aligned}
$$


where

$$
\kappa=\left(\frac{2}{2-m}\right)
$$

Substituting the boundary condition given in equation (12) into equation (16) and making use of the behavior of the modified Bessel function, we have

$$
D_{1}=0 \text {. }
$$

Substituting the continuity condition given in equation (13) into equation (16) yields

$$
\begin{aligned}
A_{1} K_{2 \kappa-1}\left(\kappa \lambda_{m} \xi_{0}^{(1 / \kappa)}\right)+B_{1} I_{2 \kappa-1}\left(\kappa \lambda_{m} \xi_{0}^{(1 / \kappa)}\right) & =C_{1} \xi_{0}^{(m / 2)} K_{1}\left(\lambda_{0} \xi_{0}\right), \\
-A_{1} K_{2 \kappa}\left(\kappa \lambda_{m} \xi_{0}^{(1 / \kappa)}\right)+B_{1} I_{2 \kappa}\left(\kappa \lambda_{m} \xi_{0}^{(1 / \kappa)}\right) & =-C_{1} \sqrt{\frac{1+i D_{s 0}}{G R\left(1+i D_{s m}\right)}} K_{2}\left(\lambda_{0} \xi_{0}\right),
\end{aligned}
$$

where $A_{1}, B_{1}$, and $C_{1}$ are undetermined constants; $G R=G_{s m} / G_{s 0}$ reflects the softening or hardening degree of the soil. $G R=1$ denotes the homogeneous soil, $G R>1$ denotes the strengthened soil, and $G R<1$ denotes the weakened soil. It is noted that, for the weakened soil, smaller GR is associated with the larger softening degree, while for the strengthened soil, greater $G R$ is corresponding to the larger hardening degree.

Combining equations (19) and (20), the following relationship between $A_{1}$ and $B_{1}$ can be determined:

$$
Q_{1}=\frac{A_{1}}{B_{1}}=\frac{\sqrt{\left(\left(1+i D_{s 0}\right) /\left(G R\left(1+i D_{s m}\right)\right)\right)} K_{2}\left(\lambda_{0} \xi_{0}\right) I_{2 \kappa-1}\left(\kappa \lambda_{m} \xi_{0}^{(1 / \kappa)}\right)+\xi_{0}^{(m / 2)} K_{1}\left(\lambda_{0} \xi_{0}\right) I_{2 \kappa}\left(\kappa \lambda_{m} \xi_{0}^{(1 / \kappa)}\right)}{-\sqrt{\left(\left(1+i D_{s 0}\right) /\left(G R\left(1+i D_{s m}\right)\right)\right)} K_{2}\left(\lambda_{0} \xi_{0}\right) K_{2 \kappa-1}\left(\kappa \lambda_{m} \xi_{0}^{(1 / \kappa)}\right)+\xi_{0}^{(m / 2)} K_{1}\left(\lambda_{0} \xi_{0}\right) K_{2 \kappa}\left(\kappa \lambda_{m} \xi_{0}^{(1 / \kappa)}\right)}
$$

The circumferential shear stress at the pile-soil interface (i.e., at $r=r_{0}$ ) can be further expressed as

$$
\begin{aligned}
\tau_{r \theta}\left(r=r_{0}\right) & =\left.\frac{G_{s m}\left(1+i D_{s m}\right)}{r_{0}}\left(\frac{\mathrm{d} u_{\theta}}{\mathrm{d} \xi}-\frac{u_{\theta}}{\xi}\right)\right|_{\xi=1} \\
& =\frac{1}{r_{0}} G_{s m}\left(1+i D_{s m}\right) \lambda_{m} \xi^{-(m / 2)+(1 / \kappa)-1} B_{1}\left[-Q_{1} K_{2 \kappa}\left(\kappa \lambda_{m} \xi^{(1 / \kappa)}\right)+I_{2 \kappa}\left(\kappa \lambda_{m} \xi^{(1 / \kappa)}\right)\right] .
\end{aligned}
$$

The torsional impedance of the soil can be defined as

$$
K_{\theta}=-\frac{2 \pi r_{0}^{3} \tau_{r \theta}\left(r=r_{0}\right)}{u_{\theta}\left(r=r_{0}\right)}=2 \pi G_{s m}\left(1+i D_{s m}\right) \lambda_{m} r_{0}^{2} \frac{Q_{1} K_{2 \kappa}\left(\kappa \lambda_{m}\right)-I_{2 \kappa}\left(\kappa \lambda_{m}\right)}{Q_{1} K_{2 \kappa-1}\left(\kappa \lambda_{m}\right)+I_{2 \kappa-1}\left(\kappa \lambda_{m}\right)},
$$

where $K_{\theta}$ is the torsional impedance of the soil.

For the convenience of subsequent analysis, $K_{\theta}$ can be further expressed as follows:

$$
K_{\theta}=G_{s 0} r_{0}^{2}\left(S_{w 1}+i S_{w 2}\right),
$$

where $S_{w 1}$ and $S_{w 2}$ are the stiffness and damping parts of the torsional impedance of the radially inhomogeneous soil, respectively.
Based on equations (16) and (21), the circumferential displacement of the solid phase at the pile-soil interface can be expressed as

$$
u_{\theta}\left(r=r_{0}\right)=B_{1}\left[Q_{1} K_{2 \kappa-1}\left(\kappa \lambda_{m}\right)+I_{2 \kappa-1}\left(\kappa \lambda_{m}\right)\right] .
$$

Substituting the continuity condition given in equation (15) into equation (25) results in 


$$
B_{1}=\frac{\phi(z) r_{0}}{Q_{1} K_{2 \kappa-1}\left(\kappa \lambda_{m}\right)+I_{2 \kappa-1}\left(\kappa \lambda_{m}\right)} .
$$

Then, the shear stress at the pile-soil interface can be further expressed as

$$
\tau_{r \theta}\left(r=r_{0}\right)=G_{s m}\left(1+i D_{s m}\right) \lambda_{m} \frac{-Q_{1} K_{2 \kappa}\left(\kappa \lambda_{m}\right)+I_{2 \kappa}\left(\kappa \lambda_{m}\right)}{Q_{1} K_{2 \kappa-1}\left(\kappa \lambda_{m}\right)+I_{2 \kappa-1}\left(\kappa \lambda_{m}\right)} \phi(z) .
$$

Substituting equation (27) into equation (11), the governing equation of the pile under time-harmonic torsional load can be rewritten as

$$
\frac{d^{2} \phi(z)}{d z^{2}}+\left[\frac{4 G_{s m}\left(1+i D_{s m}\right) \lambda_{m}}{G_{p} r_{0}^{2}} \frac{-Q_{1} K_{2 \kappa}\left(\kappa \lambda_{m}\right)+I_{2 \kappa}\left(\kappa \lambda_{m}\right)}{Q_{1} K_{2 \kappa-1}\left(\kappa \lambda_{m}\right)+I_{2 \kappa-1}\left(\kappa \lambda_{m}\right)}+\frac{\rho_{p} \omega^{2}}{G_{p}}\right] \phi(z)=0 .
$$

The general solution of ordinary differential equation (28) can be expressed as

$$
\phi(z)=\alpha_{1} \cos \left(\gamma_{1} z\right)+\alpha_{2} \sin \left(\gamma_{1} z\right)
$$

in which

$$
\gamma_{1}=\left[\frac{4 G_{s m}\left(1+i D_{s m}\right) \lambda_{m}}{G_{p} r_{0}^{2}} \frac{-Q_{1} K_{2 \kappa}\left(\kappa \lambda_{m}\right)+I_{2 \kappa}\left(\kappa \lambda_{m}\right)}{Q_{1} K_{2 \kappa-1}\left(\kappa \lambda_{m}\right)+I_{2 \kappa-1}\left(\kappa \lambda_{m}\right)}+\frac{\rho_{p} \omega^{2}}{G_{p}}\right]^{(1 / 2)},
$$

where $\alpha_{1}$ and $\alpha_{2}$ are constants to be determined by the boundary conditions.

Substituting the boundary conditions of the pile given in equation (14) into equation (29), we have

$$
\begin{aligned}
& \alpha_{1}=-\frac{T_{0}}{G_{p} I_{p} \gamma_{1} \tan \left(\gamma_{1} H-\varphi_{1}\right)}, \\
& \alpha_{2}=-\frac{T_{0}}{G_{p} I_{p} \gamma_{1}},
\end{aligned}
$$

where $\varphi_{1}=\arctan \left[\bar{k}_{\mathrm{pb}} / \gamma_{1} H\right]$ is the phase angle and $\bar{k}_{p b}=$ $\left(k_{p b} H / G_{p} I_{p}\right)$ is the dimensionless pile bottom supporting coefficient.

According to the definition of the torsional complex stiffness at the pile top proposed by Militano and Rajapakse [17], the dimensionless torsional complex stiffness $k_{T}$ at the pile top can be expressed as

$$
k_{T}=\frac{3 T_{0}}{16 G_{s 0} r_{0}^{3} \phi(z=0)}=\frac{3 \pi r_{0} \bar{\mu} \gamma_{1} \tan \left(\gamma_{1} H-\varphi_{1}\right)}{32},
$$

where $\bar{\mu}=\left(G_{p} / G_{s 0}\right)$ is the pile-soil modulus ratio.

For the convenience in the subsequent analysis, the torsional complex stiffness at the pile top can be expressed in the following dimensionless form:

$$
k_{T}=k_{T 1}+i k_{T 2}
$$

where $k_{T 1}$ is the real part of the complex stiffness at the pile top, which represents the real stiffness of the pile, and $k_{T 2}$ is the imaginary part of the complex stiffness at the pile top, which reflects the energy dissipation.
It is noted that the above solution is corresponding to the case where $m \neq 2$. When $m=2$, the denominator in equation (17) is equal to zero, and the above solution does hold. Therefore, the solution corresponding to $m=2$ is listed in Appendix A.

\section{Results and Discussion}

4.1. Torsional Impedance of the Soil. This section will mainly study the influence of soil radial heterogeneity caused by pile driving on the torsional impedance of the soil. It is worth noting that when the value of $m$ is given (see equation (2)), there is a definite relationship between $t_{m} / r_{0}$ and $G R=G_{s m} /$ $G_{s 0}$, and these two values cannot be changed arbitrarily. The soil parameters used in the following calculation are listed in Table 1.

We first compare the reduced radially inhomogeneous single-phase medium solution (i.e., dry soil solution with setting $\rho_{f}=n=0$ ) with the homogeneous single-phase solution of Novak et al. [20] to study the changing trend of soil torsional impedance corresponding to different softening and hardening degree of the soil. It can be seen from Figure 3 that, in the whole frequency range, the stiffness and damping parts of the torsional impedance (e.g., $G R=0.25$ ) corresponding to the weakened soil case are lower than those of the homogeneous medium solution by Novak et al. [20]. It is indicated that the torsional impedance of the weakened soil is remarkably lower than that of the undisturbed soil. Meanwhile, it can also be observed from Figure 3 that the stiffness and damping parts (e.g., $G R=2$ ) corresponding to the strengthened soil case are greater than those of the homogeneous medium solution by Novak et al. [20]. It means that the torsional impedance of the strengthened soil is remarkably greater than that of the undisturbed soil. Hence, it is important to consider the radial inhomogeneity of the soil when the surrounding soil is disturbed during the construction.

Figure 4 depicts the comparison of soil torsional impedance between the radially inhomogeneous saturated soil and the corresponding dry soil. It can be seen from Figure 4 that, in the entire frequency range, the torsional impedance of the saturated soil is significantly different from that of the 
TABLE 1: Calculation parameters of the saturated soil.

\begin{tabular}{lcccc}
\hline$G_{\mathrm{s} 0}(\mathrm{MPa})$ & $\rho_{\mathrm{s}}\left(\mathrm{kg} / \mathrm{m}^{3}\right)$ & $\rho_{\mathrm{f}}\left(\mathrm{kg} / \mathrm{m}^{3}\right)$ & $n$ & $k_{\mathrm{d}}(\mathrm{m} / \mathrm{s})$ \\
\hline 20 & 2650 & 1000 & 0.4 & $10^{-7}$ \\
\hline
\end{tabular}

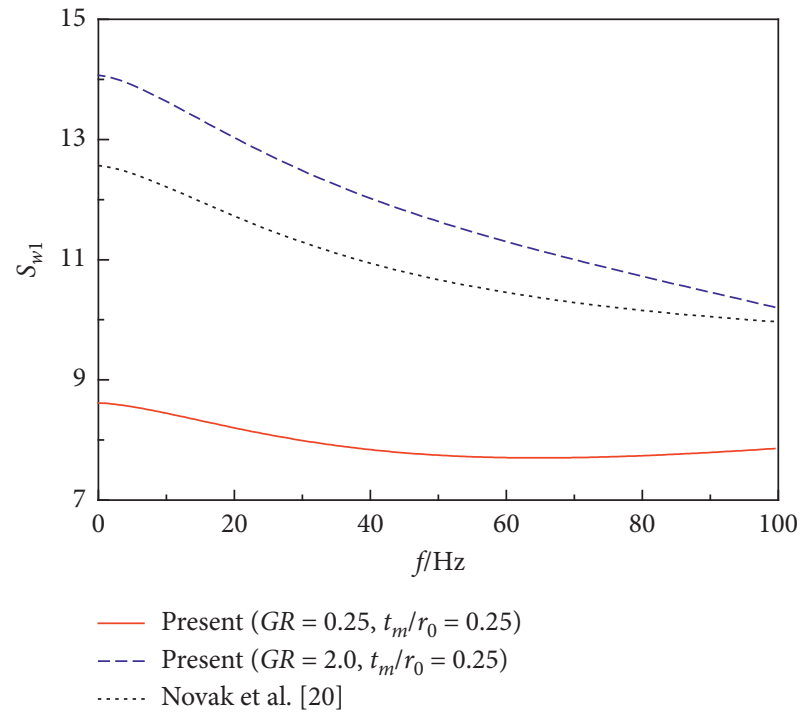

(a)

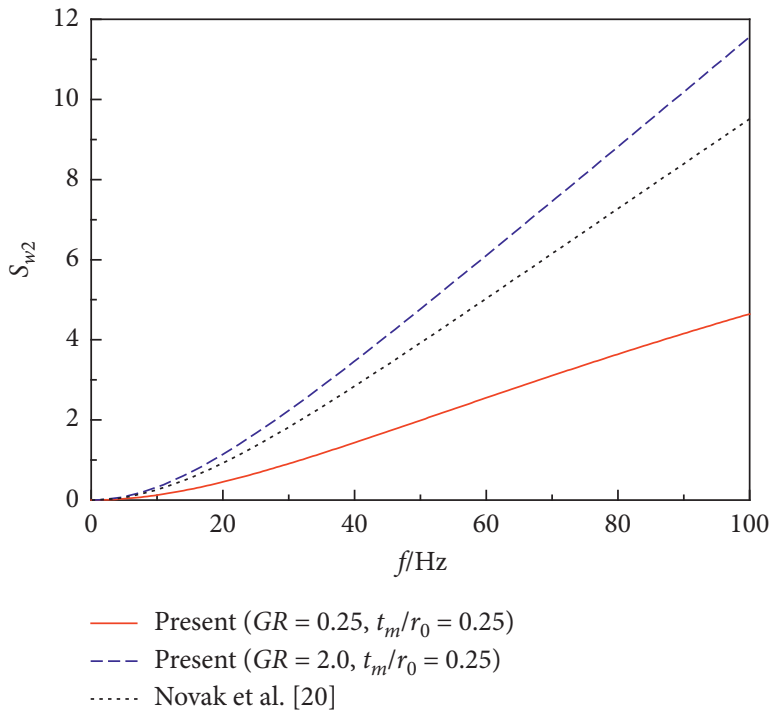

(b)

Figure 3: Comparison of the present reduced single-phase medium solution with the homogeneous medium solution $\left(D_{s m}=D_{s 0}=0\right.$, $\left.r_{0}=0.3 \mathrm{~m}\right)$.

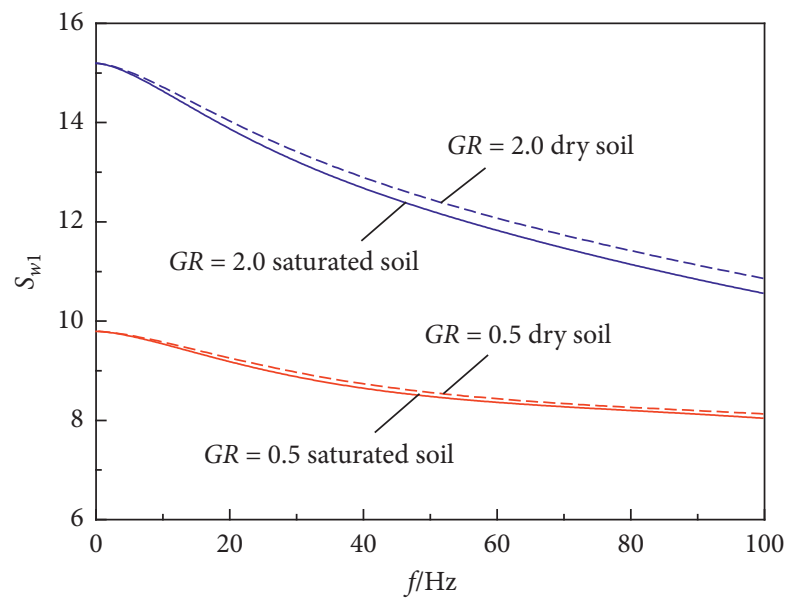

(a)

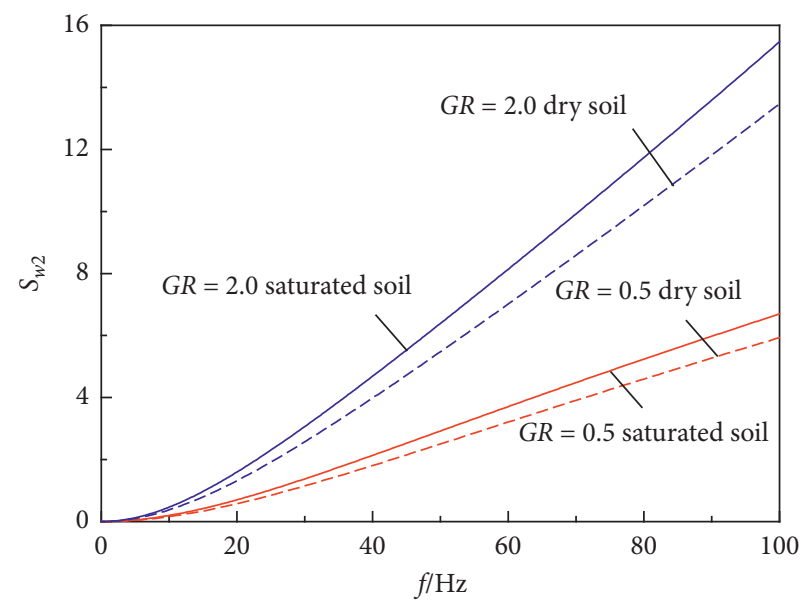

(b)

Figure 4: Comparison of torsional impedance of saturated soil solution with the reduced dry soil solution for a radially inhomogeneous soil $\left(D_{s m}=D_{s 0}=0, t_{m} / r_{0}=0.5, r_{0}=0.3 \mathrm{~m}\right)$.

dry soil, no matter it is a weakened or strengthened soil. The stiffness part of the torsional impedance of the saturated soil is lower than that of the dry soil, and the damping part of the saturated soil is greater than that of the dry soil. This indicates that the pore fluid in the saturated soil can increase the damping during the vibration process. Therefore, it is important to consider the dynamic interaction between the solid phase and the pore fluid when the voids of soil are filled with water.

4.2. Torsional Complex Stiffness at the Pile Top. This section mainly analyzes the influence of the characteristic parameters of soil radial heterogeneity on the torsional complex 


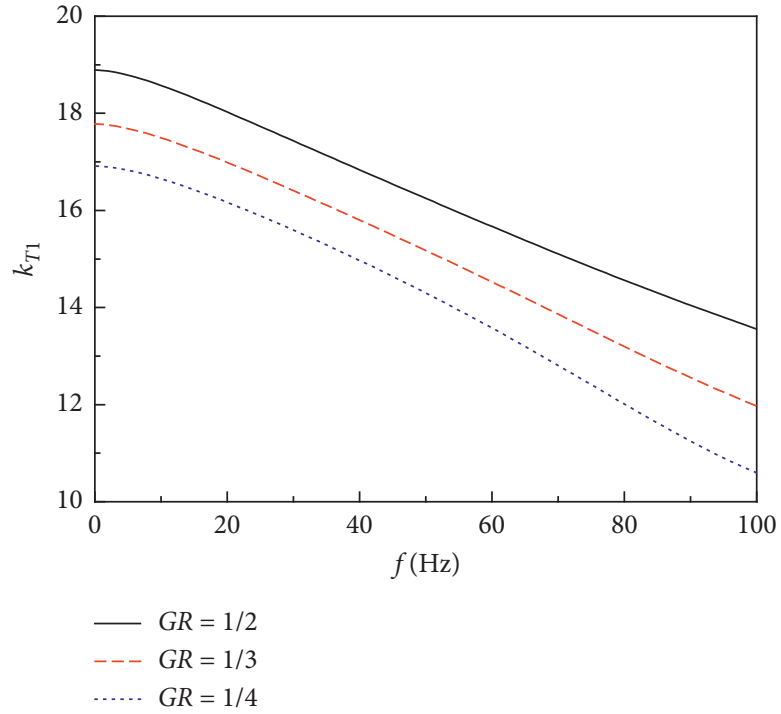

(a)

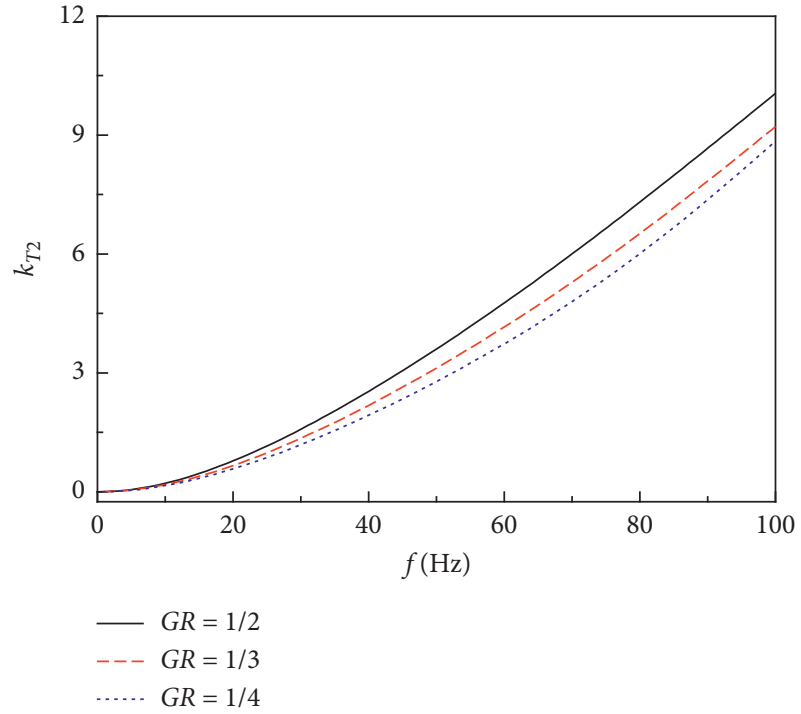

(b)

Figure 5: Influence of the shear modulus ratio $G R=G_{s m} / G_{s 0}$ on the complex stiffness at the pile top when the inner soil is weakened $\left(D_{s m}=D_{s 0}=0, t_{m} / r_{0}=0.25\right)$.

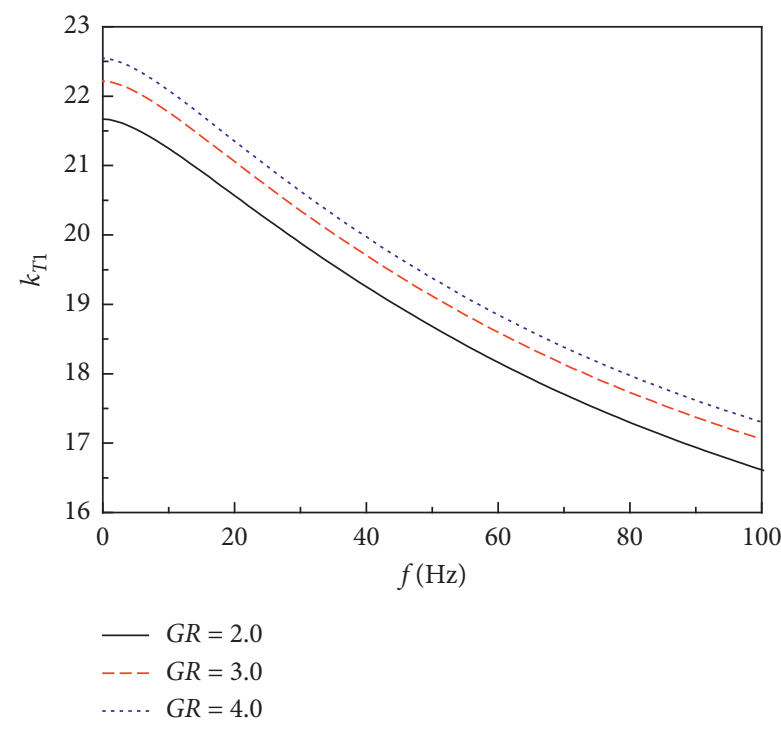

(a)

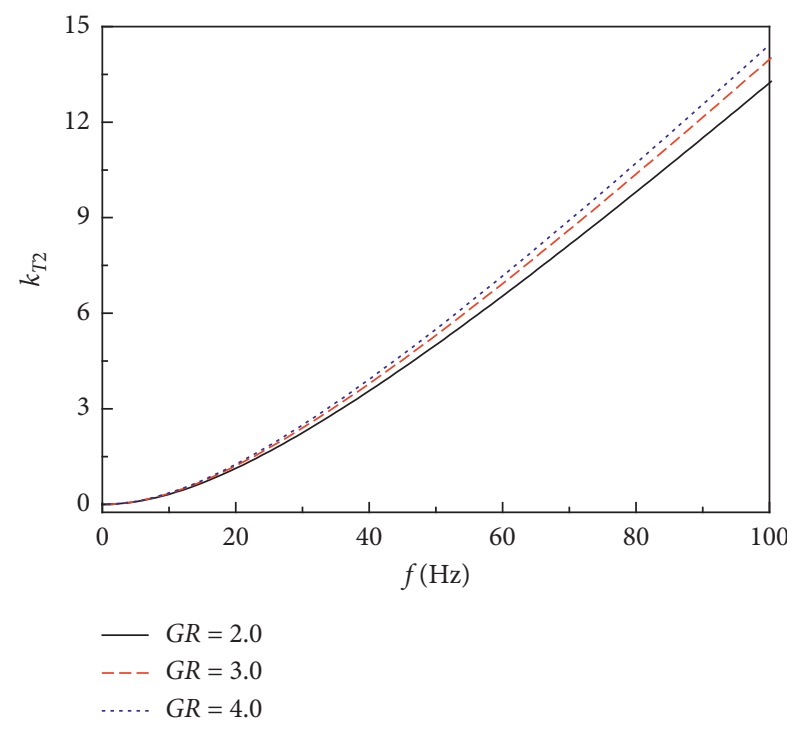

(b)

FIGURE 6: Influence of the shear modulus ratio $G R=G_{s m} / G_{s 0}$ on the complex stiffness at the pile top when the inner soil is strengthened $\left(D_{s m}=D_{s 0}=0, t_{m} / r_{0}=0.25\right)$.

stiffness at the pile top. The calculation parameters of the pile and pile bottom soil are taken as $\rho_{p}=2500 \mathrm{~kg} / \mathrm{m}^{3}$, $G_{p}=12.1 \mathrm{GPa}, H=10 \mathrm{~m}, r_{0}=0.3 \mathrm{~m}$, and $G_{s b}=20 \mathrm{MPa}$. Figure 5 describes the influence of the ratio of the shear modulus of the outer soil to that at the pile-soil interface (i.e., $G R$ ) on the complex stiffness at the pile top when the inner soil is weakened. It can be seen from Figure 5 that, in the whole frequency range, the real stiffness and dynamic damping at the pile top decrease with the increase of softening degree of the inner soil.
Figure 6 shows the influence of $G R$ on the complex stiffness at the pile top when the inner soil is strengthened. It can be seen from Figure 6 that, in the whole frequency range, the real stiffness and dynamic damping at the pile top increase with increasing GR. Compared with Figure 5, it can be observed that the real stiffness and dynamic damping corresponding to the strengthened soil are greater than those corresponding to the weakened soil, which indicates that the weakened soil can reduce the dynamic torsional resistance of the pile-soil system. Therefore, it is of great importance to 


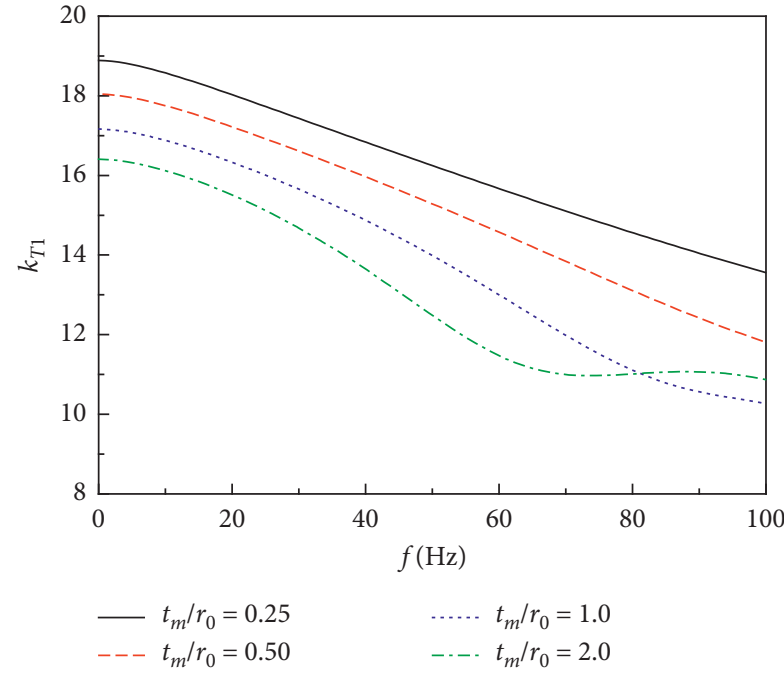

(a)

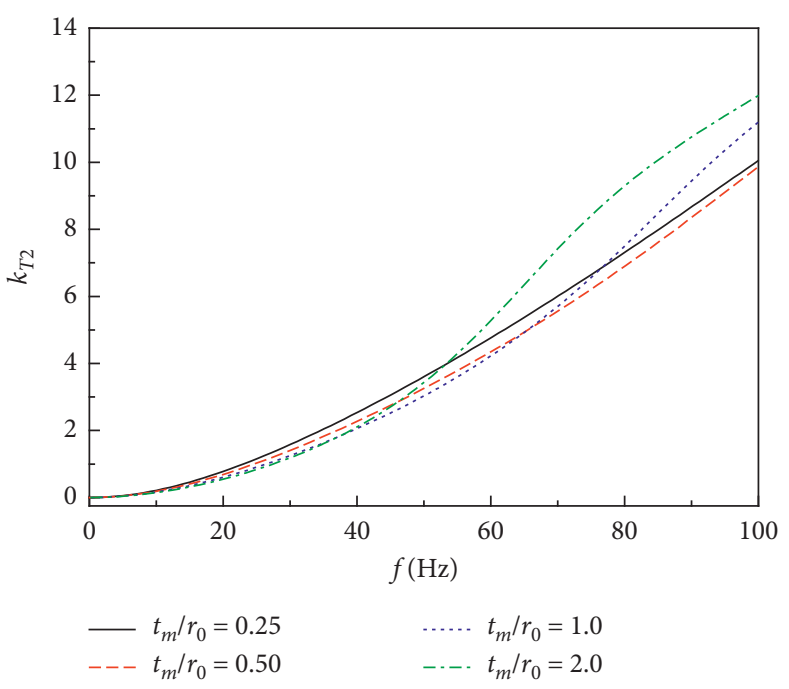

(b)

Figure 7: Influence of the disturbance range $t_{m} / r_{0}$ on the complex stiffness at the pile top when the inner soil is weakened $\left(D_{s m}=D_{s 0}=0\right.$, $G R=0.5)$.

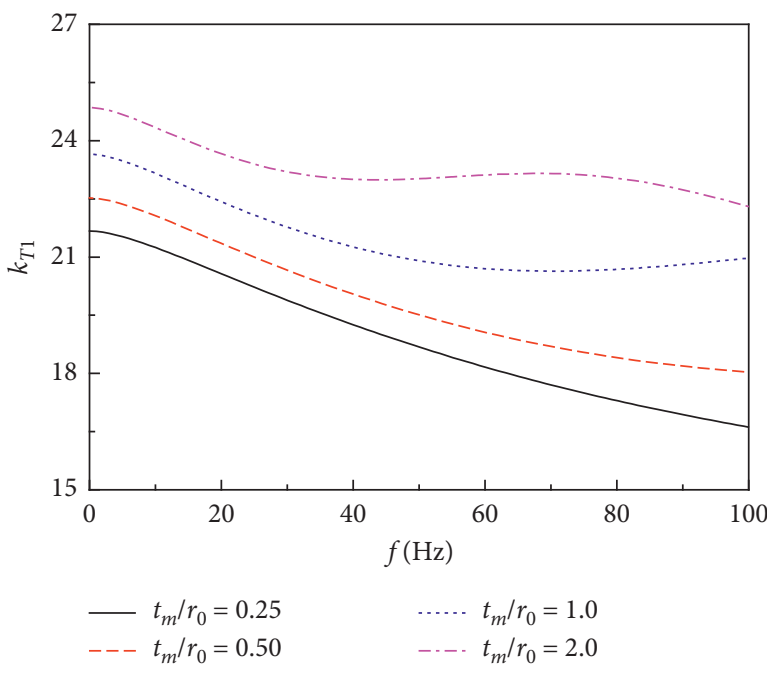

(a)

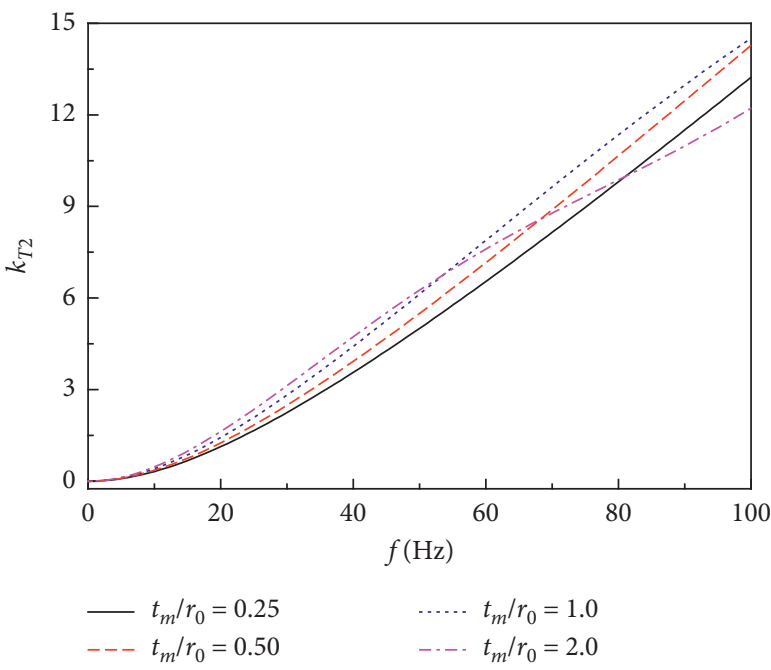

(b)

FiguRE 8: Influence of the disturbance range $t_{m} / r_{0}$ on the complex stiffness at the pile top when the inner soil is strengthened $\left(D_{s m}=D_{s 0}=0\right.$, $G R=2)$.

consider the softening degree of the inner soil in the dynamic foundation design.

Figure 7 illustrates the influence of the disturbance range on the complex stiffness at the pile top when the inner soil is weakened. It can be seen that, in the low frequency range, the real stiffness and dynamic damping at the pile top decrease with the increase of $t_{m} / r_{0}$. However, as the frequency further increases, the real stiffness and dynamic damping show certain fluctuation when $t_{m} / r_{0}$ is large (e.g., $t_{m} / r_{0}=2$ ). It can be concluded from Figure 7 that, in the low frequency range concerned in the dynamic foundation design $(0-50 \mathrm{~Hz})$, greater disturbance range will reduce the resistance of the pile-soil system to the torsional deformation for the weakened soil, which should be paid special attention in the related design.

Figure 8 shows the influence of the disturbance range on the complex stiffness at the pile top when the inner soil is strengthened. It can be seen from Figure 8 that, in the low frequency range, the real stiffness and dynamic damping at the pile top increase with the increase of $t_{m} / r_{0}$. However, when frequency is relatively high, the dynamic damping shows certain fluctuation with larger $t_{m} / r_{0}$.

Figures 9 and 10 reflect the influence of the material damping of the inner soil on the complex stiffness at the pile top corresponding to the weakened and strengthened soil cases. It can be seen that, for both weakened and 


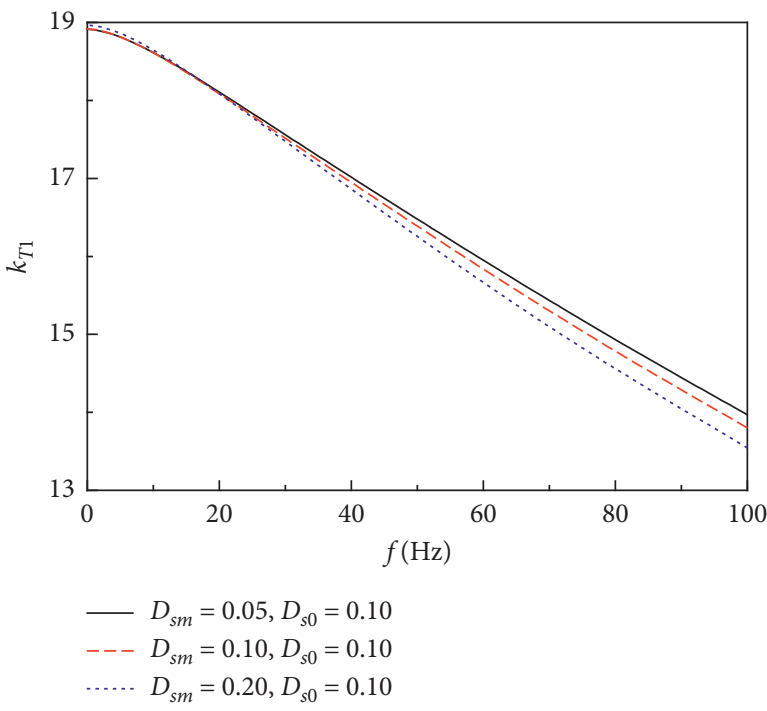

(a)

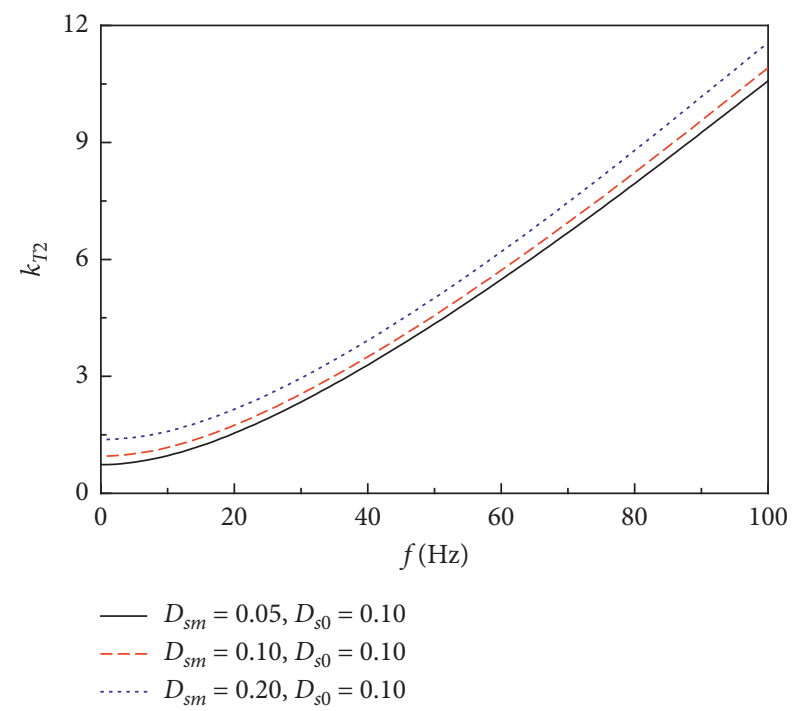

(b)

FIGURE 9: Influence of material damping of the inner disturbed soil on the complex stiffness at the pile top when the inner soil is weakened $\left(G R=0.5, t_{m} / r_{0}=0.5\right)$.

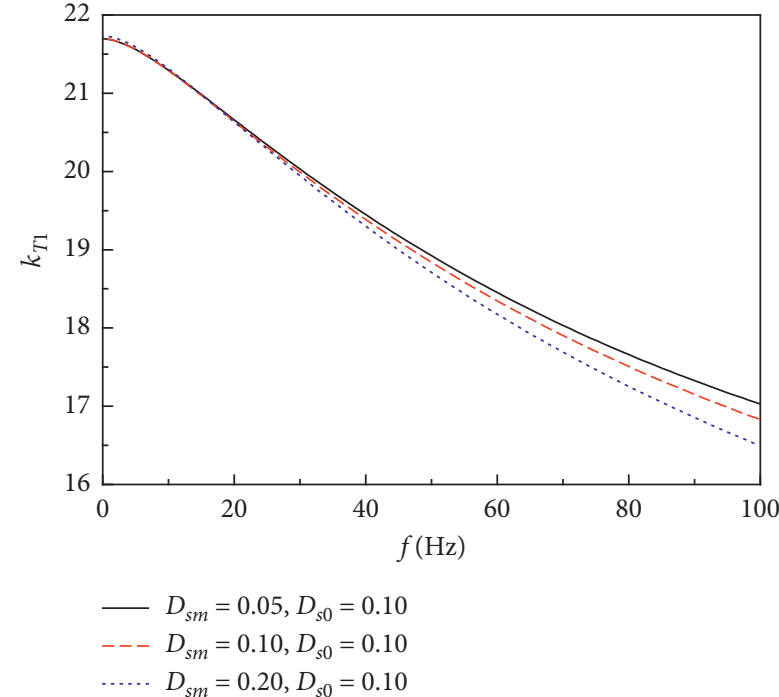

(a)

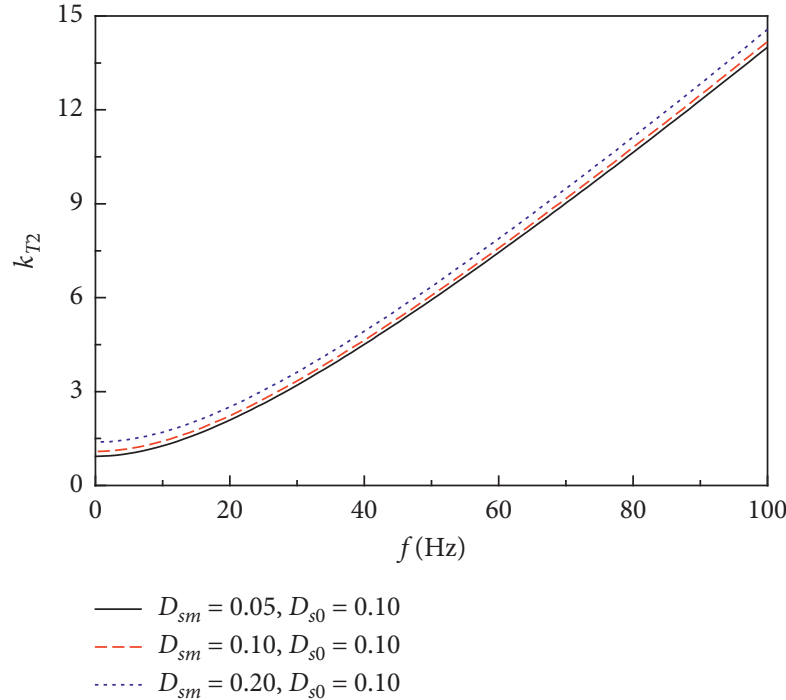

(b)

Figure 10: Influence of material damping of the inner disturbed soil on the complex stiffness at the pile top when the inner soil is strengthened $\left(G R=2, t_{m} / r_{0}=0.5\right)$.

strengthened soils, when the excitation frequency is small, the material damping of the soil in the inner region basically has negligible effect on the real stiffness. With the increase of the excitation frequency, the greater the material damping of the soil in the inner region is, the smaller the real stiffness is. Meanwhile, it can be also observed that, in the entire frequency range, the dynamic damping increases with the increase of the material damping of the inner soil. Therefore, in general, for dynamic foundation design, the damping of the soil has limited influence on the torsional complex stiffness at the pile top.

\section{Conclusions}

Based on the dynamic consolidation theory proposed by Biot, the exact solution for the torsional vibration of an elastic pile embedded in a radially inhomogeneous saturated soil with shear modulus changing in an exponential form along the radial direction is developed. This exact solution can be further served as benchmarks for future numerical methods. Through detailed calculation and analysis, it is found that when considering the dynamic interaction between the solid phase and pore fluid, the torsional 
impedance of the saturated soil is different from that of the dry soil. In addition, the softening/hardening degree and the disturbance range of the saturated soil have marked influence on the torsional complex stiffness at the pile top. However, for dynamic foundation design (i.e., the low frequency range is concerned), the damping of the soil has limited influence on the torsional complex stiffness at the pile top.

\section{Appendix}

\section{A. The Solution for the Pile-Soil System When $\boldsymbol{m}=\mathbf{2}$}

The solution of equation (9) can be expressed as

$$
\begin{aligned}
& u_{\theta}=A_{2} \xi^{\delta_{1}}+B_{2} \xi^{\delta_{2}}, \quad\left(1 \leq \xi \leq \xi_{0}\right), \\
& u_{\theta}=C_{2} K_{1}\left(\lambda_{0} \xi\right)+D_{2} I_{1}\left(\lambda_{0} \xi\right), \quad\left(\xi \geq \xi_{0}\right),
\end{aligned}
$$

where

$$
\delta_{1}=-1+\sqrt{4+\lambda_{m}^{2}} ; \delta_{2}=-1-\sqrt{4+\lambda_{m}^{2}} .
$$

Substituting the boundary condition given in equation (12) into equation (A.1) and using the properties of the modified Bessel function, we have $D_{2}=0$. Substituting the continuity condition in equation (13) into equation (A.1), we have

$$
Q_{2}=\frac{A_{2}}{B_{2}}=\frac{-\xi_{0}^{\delta_{2}}\left(\delta_{2}-1\right) K_{1}\left(\lambda_{0} \xi_{0}\right)-\lambda_{0} \xi_{0}^{\delta_{2}+1} K_{2}\left(\lambda_{0} \xi_{0}\right)}{\xi_{0}^{\delta_{1}}\left(\delta_{1}-1\right) K_{1}\left(\lambda_{0} \xi_{0}\right)+\lambda_{0} \xi_{0}^{\delta_{1}+1} K_{2}\left(\lambda_{0} \xi_{0}\right)}
$$

The circumferential shear stress amplitude at the pilesoil interface can then be derived, and the torsional impedance of the soil can be written as

$$
K_{\theta}=-\left.\frac{2 \pi r_{0}^{3} \tau_{r \theta}}{u_{\theta}}\right|_{\xi=1}=-2 \pi G_{s m}\left(1+i D_{s m}\right) r_{0}^{2} \frac{Q_{2}\left(\delta_{1}-1\right)+\left(\delta_{2}-1\right)}{Q_{2}+1} .
$$

According to the continuity conditions at the pile-soil interface, the governing equation for an elastic pile under time-harmonic torsional load can be further expressed as

$$
\frac{\mathrm{d}^{2} \phi(z)}{\mathrm{d} z^{2}}+\left\{\frac{G_{s m}\left(1+i D_{s m}\right)}{Q_{2} \xi^{\delta_{1}}+\xi^{\delta_{2}}}\left[Q_{2}\left(\delta_{1}-1\right)+\left(\delta_{2}-1\right)\right]+\frac{\rho_{p} \omega^{2}}{G_{p}}\right\} \phi(z)=0 .
$$

The solution of ordinary differential equation (A.5) can be expressed as

$$
\phi(z)=\alpha_{3} \cos \left(\gamma_{2} z\right)+\alpha_{4} \sin \left(\gamma_{2} z\right),
$$

in which

$$
\gamma_{2}=\left[\frac{G_{s m}\left(1+i D_{s m}\right)}{Q_{2} \xi^{\delta_{1}}+\xi^{\delta_{2}}}\left[Q_{2}\left(\delta_{1}-1\right)+\left(\delta_{2}-1\right)\right]+\frac{\rho_{p} \omega^{2}}{G_{p}}\right]^{(1 / 2)},
$$

where $\alpha_{3}$ and $\alpha_{4}$ are the constants to be determined by the boundary conditions.

Substituting the boundary conditions of the pile into equation (A.6), we have

$$
\begin{aligned}
& \alpha_{1}=-\frac{T_{0}}{G_{p} I_{p} \gamma_{2} \tan \left(\gamma_{2} H-\varphi_{2}\right)}, \\
& \alpha_{2}=-\frac{T_{0}}{G_{p} I_{p} \gamma_{2}},
\end{aligned}
$$

where

$$
\begin{aligned}
\varphi & =\arctan \left[\frac{\bar{k}_{p b}}{\left(\gamma_{2} H\right)}\right], \\
\bar{k}_{p b} & =\frac{k_{p b} H}{G_{p} I_{p}} .
\end{aligned}
$$

The dimensionless torsional complex stiffness $k_{T}$ at the pile top can be finally expressed as

$$
k_{T}=\frac{3 T_{0}}{16 G_{s 0} r_{0}^{3} \phi(z=0)}=\frac{3 \pi r_{0} \bar{\mu} \gamma_{2} \tan \left(\gamma_{2} H-\varphi\right)}{32} .
$$

\section{Data Availability}

The data of Figures 3-10 used to support the findings of this study are available from the corresponding author upon request.

\section{Conflicts of Interest}

The authors declare that they have no conflicts of interest.

\section{Acknowledgments}

This research was supported by the National Natural Science Foundation of China (Grant no. 52078467), Natural Science Foundation of Zhejiang Province (Grant no. LHZ21E090001), and Research and Development Fund of Zhejiang A \& F University (Grant no. 2020FR052).

\section{References}

[1] E. Pan, H. Liu, and Z. Zhang, "Vertical and torsional vibrations of a rigid circular disc on a transversely isotropic and layered half-space with imperfect interfaces," Soil Dynamics and Earthquake Engineering, vol. 113, pp. 442-453, 2018.

[2] Z. Zhang and E. Pan, "Vertical and torsional vibrations of an embedded rigid circular disc in a transversely isotropic multilayered half-space," Engineering Analysis with Boundary Elements, vol. 99, pp. 157-168, 2019.

[3] R. Y. S. Pak and F. Abedzadeh, "Forced torsional oscillation from the interior of a half-space," Journal of Sound and Vibration, vol. 160, no. 3, pp. 401-415, 1993.

[4] Y. Le, N. Wang, W. T. Hu, D. X. Geng, and Y. L. Jiang, "Torsional dynamic impedance of a stepped pile based on the wedged soil model," Computers and Geotechnics, vol. 128, Article ID 103854, 2020. 
[5] W. Wu, H. Liu, M. H. El Naggar, G. Mei, and G. Jiang, "Torsional dynamic response of a pile embedded in layered soil based on the fictitious soil pile model," Computers and Geotechnics, vol. 80, pp. 190-198, 2016.

[6] L. G. Tham, Y. K. Cheung, and Z. X. Lei, "Torsional dynamic analysis of single piles by time-domain BEM," Journal of Sound and Vibration, vol. 174, no. 4, pp. 505-519, 1994.

[7] M. Novak and M. Sheta, Approximate Approach to Contact Problems of Piles, Proceedings of the Geotechnical Engineering Division, American Society of Civil Engineering National Convention, Florida, USA, 1980.

[8] A. S. Veletsos and K. W. Dotson, "Impedances of soil layer with disturbed boundary zone," Journal of Geotechnical Engineering, vol. 112, no. 3, pp. 363-368, 1986.

[9] A. S. Veletsos and K. W. Dotson, "Vertical and torsional vibration of foundations in inhomogeneous media," Journal of Geotechnical Engineering, vol. 114, no. 9, pp. 1002-1021, 1988.

[10] K. W. Dotsos and A. S. Veletsos, "Vertical and torsional impedances for radially inhomogeneous viscoelastic soil layer," Soil Dynamics and Earthquake Engineering, vol. 9, no. 3, pp. 110-119, 1990.

[11] Y. C. Han and G. C. Sabin, "Impedances for radially inhomogeneous viscoelastic soil media," Journal of Engineering Mechanics, vol. 121, no. 9, pp. 939-947, 1995.

[12] Z. Li and Y. F. Gao, "Torsional vibration of a large-diameter pipe pile embedded in inhomogeneous soil," Ocean Engineering, vol. 172, pp. 737-758, 2019.

[13] Z. Zhang and E. Pan, "Dynamic torsional response of an elastic pile in a radially inhomogeneous soil," Soil Dynamics and Earthquake Engineering, vol. 99, pp. 35-43, 2017.

[14] M. A. Biot, "Theory of propagation of elastic waves in a fluidsaturated porous solid. I. Low-Frequency range," The Journal of the Acoustical Society of America, vol. 28, no. 2, pp. 168-178, 1956.

[15] M. A. Biot, "Mechanics of deformation and acoustic propagation in porous media," Journal of Applied Physics, vol. 33, no. 4, pp. 1482-1498, 1962.

[16] Z. Li, Y. Gao, and K. Wang, "Torsional vibration of an end bearing pile embedded in radially inhomogeneous saturated soil," Computers and Geotechnics, vol. 108, pp. 117-130, 2019.

[17] G. Militano and R. K. N. D. Rajapakse, "Dynamic response of a pile in a multi-layered soil to transient torsional and axial loading," Géotechnique, vol. 49, no. 1, pp. 91-109, 1999.

[18] O. C. Zienkiewicz, C. T. Chang, and P. Bettess, "Drained, undrained, consolidating and dynamic behaviour assumptions in soils," Géotechnique, vol. 30, no. 4, pp. 385-395, 1980.

[19] Z. Zhang and E. Pan, "Time-harmonic response of transversely isotropic and layered poroelastic half-spaces under general buried loads," Applied Mathematical Modelling, vol. 80, pp. 426-453, 2020.

[20] M. Novak, T. Nogami, and A. E. Fakhry, "Dynamic soil reactions for plane strain case," Journal of the Engineering Mechanics Division, ASCE, EM4, vol. 104, pp. 953-959, 1978. 